\title{
Deconfinement criticality for the spatially anisotropic triangular antiferromagnet with the ring exchange
}

\author{
Yoshihiro Nishiyama \\ Department of Physics, Faculty of Science, \\ Okayama University, Okayama 700-8530, Japan
}

(Dated: November 29, 2018)

\begin{abstract}
The spatially anisotropic triangular antiferromagnet is investigated with the numerical diagonalization method. As the anisotropy varies, the model changes into a variety of systems such as the one-dimensional, triangular, and square-lattice antiferromagnets. Taking into account such a geometrical character, we impose the screw-boundary condition, which interpolates smoothly the oneand two-dimensional lattice structures. Diagonalizing the finite clusters with $N=16,20, \ldots, 32$ spins, we observe an intermediate phase between the VBS and Néel phases. Suppressing the intermediate phase by applying the ring exchange, we realize a direct VBS-Néel transition. The simulation data indicate that the transition is a continuous one with the correlation-length critical exponent $\nu=0.80(15)$. These features are in agreement with the deconfinement-criticality scenario advocated by Senthil and coworkers in the context of the high-temperature superconductivity.
\end{abstract}




\section{INTRODUCTION}

According to the deconfinement-criticality scenario,,$\frac{1,2,3,4}{2}$ in $(2+1)$ dimensions, the phase transition separating the VBS and Néel phases is continuous, accompanied with unconventional critical indices. Naively, such a transition should be discontinuous, $\underset{2}{2}$ because the adjacent phases possess distinctive order parameters such as the VBS-coverage pattern, and the sublattice magnetization, respectively. A good deal of field-theoretical investigations have been made to clarify this issue. ${ }^{5} \cdot \underline{6}$ For instance, as a low-energy effective description, the $\mathrm{QED}_{3}$ theory has been investigated, ${ }^{7}, \underline{8}, \underline{9}$ it would be intriguing that the theory exhibits a deconfinement transition. 10 On the one hand, because of the magnetic frustration, the Monte Carlo simulation suffers from the negative-sign problem to realize the VBS phase. However, in recent Monte Carlo simulations, $\stackrel{11,12}{1}$ the biquadratic interaction, rather than the magnetic frustration, has been utilized. Thereby, it turned out that the biquadratic-interaction-driven transition is a continuous one with unconventional critical indices. (On the contrary, in Refs. 13, 14, 15, it was claimed that the transition would be a weak first-order one.)

In this paper, we investigate the spatially anisotropic triangular antiferromagnet $\underline{16}, \underline{17}$ with the ring exchange by means of the numerical diagonalization method. As the anisotropy varies, the model changes into the one- and two-dimensional systems, and correspondingly, a variety of phases appear. To cope with such a geometrical peculiarity, we impose the screw-boundary condition, which interpolates the one- and two-dimensional lattice structures smoothly.

To be specific, we present the Hamiltonian for the spatially anisotropic triangular antiferromagnet with the ring exchange;

$$
\mathcal{H}=J \sum_{\langle i j\rangle} \mathbf{S}_{i} \cdot \mathbf{S}_{j}+J^{\prime} \sum_{\langle\langle i j\rangle\rangle} \mathbf{S}_{i} \cdot \mathbf{S}_{j}+J_{4} \sum_{[i j k l]}\left(P_{i j k l}+P_{i j k l}^{-1}\right)
$$

The quantum spin-1/2 operators $\left\{\mathbf{S}_{i}\right\}$ are placed at each triangular-lattice point $i$. The symbol $P_{i j k l}$ denotes a ring-exchange operator with respect to a plaquette $[i, j, k, l]$ consisting of two adjacent triangles; namely, as to a plaquette state $\left|S_{S_{3}}^{S_{1}} \square_{S_{4}}^{S_{2}}\right\rangle$, the operation $P_{1234}$ trans-

lates it into $\left.\left.\right|_{S_{4}} ^{S_{3}} \square_{S_{2}}^{S_{1}}\right\rangle$. The summations $\sum_{\langle i j\rangle}, \sum_{\langle\langle i j\rangle\rangle}$, and $\sum_{[i j k l]}$ run over all possible vertical nearest-neighbor pairs, remaining nearest-neighbor pairs, and plaquette spins, respectively; the triangular lattice is directed so that one of the triangular edges points upward. The parameters $J, J^{\prime}$, and $J_{4}$ are the corresponding coupling constants. (In the next section, we 
present an explicit expression for the Hamiltonian matrix, referring to the technical details of the screw-boundary condition.) Hereafter, we consider $J$ as a unit of energy; namely, we set $J=1$.

In Fig. 1, we present a schematic phase diagram; the details are explained in Sec. III, As mentioned above, the aim of this paper is to survey the direct VBS-Néel transition; in this sense, the ring exchange $J_{4}$ is significant to realize the VBS-Néel transition. A number of limiting cases were studied in Refs. 18, 19, 20, 21, 22, 23: First, the case $J_{4}=$ 0 was investigated with the spin-wave, $\frac{18}{,}$ series-expansion, $, \underline{19}, 20$ large- $N, \stackrel{21}{,}$ and numericaldiagonalization $^{22}$ methods. The regime of the intermediate (triangular antiferromagnetic) phase $^{24}$ was estimated as $0.27<J^{\prime}<2,0.25<J^{\prime}<1.43,0.13<J^{\prime}<1.71$, and $0.78(5)<$ $J^{\prime}<1.15(10)$, respectively. (Some analyses predict two types of intermediate phases. Such a detail is ignored for simplicity.) These results appear to be unsettled. It is a purpose of this paper to survey the intermediate phase. Second, the spatially isotropic $\left(J^{\prime}=J\right)$ case in the presence of the ring exchange was investigated in Ref. 23; here, the generic types of ring-exchange interactions were considered in the context of the Helium adsorbate. It was reported that the $J_{4}$-driven phase transition occurs in agreement with our observation.

As mentioned above, the model (1) has a geometrical peculiarity. That is, as the spatial anisotropy $J^{\prime}$ changes, the model (1) reduces to the one-dimensional $\left(J^{\prime}=0\right)$, triangular $\left(J^{\prime}=1\right)$, and square-lattice $\left(J^{\prime} \rightarrow \infty\right)$ antiferromagnets successively. (Hence, for sufficiently large $J^{\prime}$, the conventional non-collinear Néel phase appears.) Notably enough, the phase diagram, Fig. 1, reflects this geometrical character. In order to take into account this geometrical character, we implemented the screw-boundary condition, which interpolates the one- and two-dimensional-lattice structures smoothly.

In fairness, it has to be mentioned that the VBS-Néel transition was studied for the frustrated square-lattice antiferromagnet, namely, the $J_{1}-J_{2}$ model ${ }^{25,26,27}$ According to the series-expansion method,, 25 the Néel $\left(J_{2} / J_{1} \lesssim 0.4\right)$, VBS $\left(0.4 \lesssim J_{2} / J_{1} \lesssim 0.6\right)$, and collinear $\left(0.6 \lesssim J_{2} / J_{1}\right)$ phases appear successively, as the magnetic frustration changes. The VBS phase seems to be dominated by the presence of the collinear phase. (Note that for $J_{2} / J_{1} \rightarrow$ $\infty$, the system reduces to two independent square-lattice antiferromagnets. The collinear state consists of two independent Néel orders.) In this paper, we dwell on the triangular antiferromagnet (11), which exhibits an isolated VBS-Néel transition.

The rest of this paper is organized as follows. In Sec. III, we explicate the simulation 
algorithm, placing an emphasis on the screw-boundary condition. In Sec. III, we show the finite-size-scaling analysis of the simulation data. In Sec. IV, we present the summary and discussions.

\section{SIMULATION METHOD: SCREW-BOUNDARY CONDITION}

In this section, we present an explicit expression for the Hamiltonian, Eq. (1), under the screw-boundary condition.

To begin with, we present a schematic drawing of the finite-size cluster in Fig. 2. As shown in the figure, the spins constitute a one-dimensional $(d=1)$ alignment $\left\{\mathbf{S}_{i}\right\}(i=1,2, \ldots, N)$. The dimensionality is lifted to $d=2$ by the bridges over the $v$ th-neighbor interactions. As mentioned in the Introduction, the spatially anisotropic triangular antiferromagnet possesses a geometrical character such that it reduces to a one-dimensional antiferromagnet in the limit $J^{\prime} \rightarrow 0$. In this sense, the geometrical peculiarity is seized by the screw-boundary condition. Actually, for a rectangular cluster with the system size $6 \times 6$, for instance, the length of the independent chains in the limit $J^{\prime} \rightarrow 0$ is merely $L=6$. On the contrary, owing to the screw-boundary condition, we attain treating the chain length $L=32$ along the $J$-bond direction.

To be specific, we present an explicit expression for the Hamiltonian matrix. We propose the following expression;

$$
\mathcal{H}=J H(1)+J^{\prime}(H(v)+H(v+1))+J_{4}\left(H_{4}(1, v)+H_{4}(1, v+1)+H_{4}(v+1, v)\right) .
$$

Here, the $v$ th-neighbor Heisenberg interaction $H(v)$ is given by

$$
H(v)=\sum_{i=1}^{N} \mathbf{S}_{i} \cdot \mathbf{S}_{i+v} .
$$

(The periodic condition, namely, $\mathbf{S}_{N+i}=\mathbf{S}_{i}$, is imposed.) Similarly, the ring exchange is introduced via

$$
H_{4}(j, v)=\sum_{i=1}^{N}\left(P_{i, i+j, i+v, i+j+v}+\text { h.c. }\right) .
$$

We set the screw pitch to

$$
v(N)= \begin{cases}n(\sqrt{N})+1 & \text { for } N \geq 24 \\ n(\sqrt{N}) & \text { otherwise }\end{cases}
$$


with the round-off function $n(x)=[x+0.5]$ and Gauss' symbol [...]; i.e., $n(2.4)=2$. The screw pitch $v(N)$ converges to $v(N) / \sqrt{N} \rightarrow 1$ for large system sizes $N \rightarrow \infty$; hence, the spins form a $\sqrt{N} \times \sqrt{N}$ network embedded on the torus. The rule, Eq. (5), is intended to suppress the finite-size errors; actually, by Eq. (15), we can set the screw-pitch $v$ to an even number (for small $N$ ), which turns out to improve the finite-size behavior even for small system sizes. More specifically, the screw-boundary condition introduces a frustration particularly for the Néel-type magnetism $\left(J^{\prime} \rightarrow \infty\right)$, and the frustration effect is suppressed by the above rule, Eq. (15).

The above formulae complete the basis of our scheme. As shown in Fig. 2, the embedding geometry under the screw-boundary condition is essentially one-dimensional, admitting us to calculate the Hamiltonian-matrix elements systematically with Eq. (2). In the next section, utilizing the Lanczos method, we diagonalize the Hamiltonian matrix for the system sizes $N \leq 32$.

\section{NUMERICAL RESULTS}

In this section, we present the numerical results. We calculate the excitation gap

$$
\Delta E_{i}\left(k, S_{\text {tot }}^{z}\right)=E_{i}\left(k, S_{\text {tot }}^{z}\right)-E_{0}\left(0,0^{+}\right)
$$

with the $i$ th low-lying energy $E_{i}\left(k, S_{\text {tot }}^{z}\right)(i=1,2, \ldots)$ within the sector $\left(k, S_{\text {tot }}^{z}\right)$. Here, the index $k$ denotes the wave number within the Brillouin zone $-\pi \leq k \leq \pi$. We impose the screw-boundary condition (Fig. 2), and the Bloch wave $k$ extends along the spiral (J-bond) chain; hence, the reciprocal space is one-dimensional. The quantum number $S_{\text {tot }}^{z}$ denotes

an eigenvalue of the operator $\sum_{i=1}^{N} S_{i}^{z}$. In the case of $S_{t o t}^{z}=0$, additionally, we introduce an index \pm , which specifies the inversion symmetry with respect to $S_{i}^{z} \rightarrow-S_{i}^{z}$. The sector $\left(0,0^{+}\right)$contains the ground state. In this sector, we shift the $i$ index so as to express the ground-state energy as $E_{0}\left(0,0^{+}\right)$via $i \rightarrow i-1$. (The ground-state energy is the starting point of all excitations, and it is sensible to index the ground-state energy as $E_{i=0}$ rather than $i=1$.) The linear dimension $L$ of the cluster is given by

$$
L=\sqrt{N}
$$

because the $N$ spins constitute a two-dimensional network as shown in Fig. 2 , 


\section{A. Spatially anisotropic triangular antiferromagnet: $J_{4}=0$}

In this section, we survey the regime without the ring exchange $J_{4}=0$; as mentioned in the Introduction, this case has been studied in Refs. 18, 19,20,21,22, and the details of the intermediate phase remain unclear.

In Fig. 3, we plot the excitation gap $\Delta E_{1}\left(\pi, 0^{+}\right)$for $J_{4}=0$, various $J^{\prime}$ and $N=$ $16,20, \ldots, 32$. We notice that the level crossings take place at $J^{\prime} \approx 0.65$ and $J^{\prime} \approx 1.1$. That is, the softening instability, $\Delta E_{1}\left(\pi, 0^{+}\right)<0$, occurs in the intermediate regime. We estimate the range of the intermediate phase as

$$
0.65(15)<J^{\prime}<1.1(1)
$$

Here, as an error indicator, we utilize the data scatters of the $J^{\prime}$-intercept among $N=20$, 24, 28, and 32. (Several related studies are overviewed afterward.)

Surveying various parameter ranges, we found that the elementary-excitation gap opens at either $k=0$ or $\pi$. The softening of the branch $k=\pi$ suggests that the magnetic order along the $J$-bond direction is unstable against a staggered modulation. Such a staggered modulation fits the boundary condition (constraint) such that the chain length $N$ is always set to an even number. On the one hand, as shown in Fig. 2, the number of spiral turns, $N / v$, of the chain is a fractional number, and the magnetism along the spiral direction may not fit the embedding geometry. Hence, the staggered order along the chain direction becomes even stabilized, resulting in the $k=\pi$ softening. On the one hand, in the VBS phase $J^{\prime}<0.65$, the energy gap $\Delta E_{1}\left(\pi, 0^{+}\right)$gets closed as the system size enlarges; eventually, the ground state may be doubly degenerated in the thermodynamic limit. This double degeneracy suggests that the $J$-bond chain is covered by the dimers. (In this sense, the VBS picture of the present system is not so complicated, as compared to that of the square lattice. $\stackrel{28,29}{2}$ )

On the contrary, in the Néel phase $1.1<J^{\prime}$, a positive gap $\Delta E_{1}\left(\pi, 0^{+}\right)>0$ starts to open. In fact, in the limit $J^{\prime} \rightarrow \infty$, the model reduces to the square-lattice antiferromagnet. Hence, the spins along the diagonal ( $J$-bond) direction align ferromagnetically, and the $k=\pi$ excitation exhibits a mass gap.

It is a good position to make an overview of the related studies. According to the

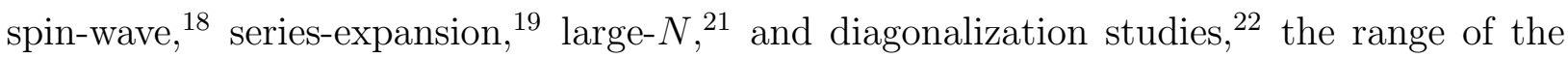
intermediate phase is estimated as $0.27<J^{\prime}<2,0.25<J^{\prime}<1.43,0.13<J^{\prime}<1.71$, 
and $0.78(5)<J^{\prime}<1.15(10)$, respectively. Our result, Eq. (8) , indicates that the VBS phase persists up to a considerably large $J^{\prime}$, suggesting that the VBS phase is robust. Similar conclusion was drawn from the diagonalization study by Weng and coworkers. ${ }^{22}$ They diagonalized the rectangular clusters with the sizes $6 \times 4,8 \times 4$, and $6 \times 6$. Such a rectangular geometry is suitable for investigating the Néel-type magnetic structure. On the contrary, the screw-boundary condition meets the quasi-one-dimensional system (VBS phase). The agreement between these approaches would be encouraging.

As a reference, in Fig. 4, we present the ground-state energy per unit cell, $E_{0}\left(0,0^{+}\right) / N$, with $N=32$ for the same parameter range as that of Fig. 3, In the small- $J^{\prime}$ regime, the ground-state energy is close to the Bethe-ansatz solution, $E_{0} / N=-0.443 \ldots$, for the one-dimensional Heisenberg antiferromagnet. This fact suggests that the VBS phase is of one-dimensional character.

Last, we mention a number of remarks concerning the phase diagram. We made similar analyses for various values of $J_{4} \sim 0$, The result is summarized in Fig. 1, as suggested by Eq. (8), the intermediate-phase boundaries are not determined very precisely, and the boundaries in Fig. 1 are only schematic. (The critical branch separating the VBS and Néel phases is considered in the next section.) Concerning the singularities of the phase boundaries surrounding the intermediate phase, we followed the claim 22 that the intermediate-VBS (-Néel) phase transition is discontinuous (continuous).

\section{B. Suppression of the intermediate phase by the ring exchange $J_{4}$}

In this section, we explore the regime with the ring exchange $J_{4} \neq 0$.

In Fig. 5, we plot the excitation gap $\Delta E_{1}\left(\pi, 0^{+}\right)$for $J_{4}=0.07$, various $J^{\prime}$ and $N=$ $16,20, \ldots, 32$. Comparing the result with that of Fig. 3. we notice that the ring exchange $J_{4}$ suppresses the intermediate phase (softening instability). As mentioned in the Introduction, the suppression of the intermediate phase by $J_{4}$ was demonstrated in Ref. 23 at $J^{\prime}=1$. In the present study, we have yet another parameter $J^{\prime}$, and we are able to investigate the $J^{\prime}$-driven VBS-Néel transition.

In Fig. 6, we plot the scaled energy gap $L^{z} \Delta E_{1}(0,1)$ for $J_{4}=0.07$, various $J^{\prime}$, and $N=16,20, \ldots, 32$. Note that the sector $S_{t o t}^{z}=1$ corresponds to the triplet excitation created preferentially on the $J$-bond chain. The behavior of the triplet excitation contains 
information on the phase transition from the VBS phase. Here, we set the dynamical critical exponent to $z=1$, following the conclusion of the Monte Carlo analyses 11,12 for the squarelattice antiferromagnet. (Note that the energy gap $\Delta E_{1}$ is proportional to the reciprocal correlation length, and the product $L \Delta E_{1}$ is a dimensionless (scale invariant) quantity.) According to the scaling theory, the curves of the scaled energy gap should intersect each other at the critical point. In fact, we observe that a phase transition takes place at $J^{\prime}=1.2$. Surveying various parameter ranges, we arrive at the phase diagram, as depicted in Fig. 1.

A remark is in order. As mentioned in the Introduction, naively, the VBS-Néel transition should be discontinuous; $\stackrel{2}{-}$ actually, the adjacent phases possess distinctive order parameters such as the dimer-coverage pattern and the sublattice magnetization, respectively. However, according to the deconfinement-criticality scenario, $\frac{1}{-}$ the spinon deconfining from a sea of singlet dimers changes the singularity to a continuous one. Our result supports this scenario. In the next section, surveying a critical index, we investigate the criticality of the VBS-Néel transition more in detail.

\section{Deconfinement criticality}

In this section, we estimate the critical exponent $\nu$ for the VBS-Néel transition.

In Fig. [7, we present the finite-size-scaling plot, $\left(J^{\prime}-J_{c}^{\prime}\right) L^{1 / \nu}-L \Delta E_{1}(0,1)$, for $J_{4}=0.07$, various $J^{\prime}$, and $N=16,20, \ldots, 32$. Here, we set the scaling parameters to $J_{c}^{\prime}=1.2$ and $\nu=0.8$; note that the former parameter $J_{c}^{\prime}=1.2$ was determined in Fig. 6. The data of Fig. 7 collapse into a scaling curve, confirming that the transition is indeed critical. Moreover, the critical exponent acquires an enhancement, as compared to that of the $3 d$ Heisenberg universality, $\nu=0.7112(5) \cdot \frac{32}{}$ (An overview of the related studies is addressed afterward.) Similarly, in Fig. 8, we present the finite-size-scaling plot, $\left(J^{\prime}-J_{c}^{\prime}\right) L^{1 / \nu}-L \Delta E_{1}(0,1)$, for $J_{c}^{\prime}=1.4, \nu=0.8, J_{4}=0.1$, and $N=16,20, \ldots, 32$. Again, the data collapse satisfactorily. Surveying various parameter ranges, we arrive at

$$
\nu=0.80(15)
$$

This is a good position to make an overview of the preceding Monte Carlo studies. As for the square-lattice antiferromagnet, the biquadratic-interaction-driven VBS-Néel transition

was investigated in Refs. 11 and 12 , and the critical exponent was estimated as $\nu=0.78(3)$ 
and $\nu=0.68(4)$, respectively. Moreover, as for the quasi-one-dimensional spin-1 antiferromagnet, the index $\nu=1 / 2.9$ was reported; ${ }^{30}$ see Ref. 31 for a field-theoretical interpretation. (Note that these models are free from the negative-sign problem, and the quantum Monte Carlo method is applicable.) We notice that the results are not quite settled. A notable point is that the exponent $\frac{11}{2} \nu=0.78(3)$ is significantly larger than that of the $d=3$ Heisenberg universality class $\nu=0.7112(5), \frac{32}{2}$ suggesting a peculiarity of the deconfinement criticality. Our result, Eq. (9), also suggests a tendency of an enhancement as to $\nu$. Nevertheless, our simulation result provides an evidence that the VBS-Néel transition is a critical one in agreement with the deconfinement-criticality scenario advocated by Senthil and coworkers.

\section{SUMMARY AND DISCUSSIONS}

The spatially anisotropic triangular antiferromagnet with the ring exchange, Eq. (11), was investigated by means of the numerical diagonalization method. As the spatial anisotropy $J^{\prime}$ varies, the model changes into a variety of systems such as the one-dimensional, triangular, and square-lattice antiferromagnets successively. Taking into account such a geometrical character, we adopt the screw-boundary condition, as shown in Fig. 2,

First, we survey the regime without the ring exchange $J_{4}=0$. The simulation result indicates that the intermediate phase appears in $0.65(15)<J^{\prime}<1.1(1)$. Our result shows that the VBS phase is robust 20,22 against the interchain coupling $J^{\prime}$. Second, by applying the ring exchange $J_{4}$, we suppress the intermediate phase. Eventually, we attain the direct VBS-Néel transition, which is under the current theoretical interest in the context of the high-temperature superconductivity. Postulating $z=1, \underline{11,12}$ we analyze the simulation data in terms of the finite-size scaling. Thereby, we estimate the correlation-length critical exponent as $\nu=0.80(15)$, confirming that the VBS-Néel transition is indeed a critical one. The exponent is comparable to the preceding Monte Carlo results, $\nu=0.78(3) \underline{11}$ and $\nu=0.68(4), \frac{12}{2}$ calculated for the square-lattice antiferromagnet.

Our result provides an evidence that the VBS-Néel transition is critical, realizing the deconfinement criticality. Here, the ring exchange plays a significant role. In Ref. 23, generic types of ring-exchange interactions are considered in the context of the Helium adsorbate. Such an extension may also lead to an improvement as to the finite-size behavior. This problem will be addressed in a future study. 


\section{Acknowledgments}

This work was supported by a Grant-in-Aid from Monbu-Kagakusho, Japan (No. 18740234).

1 T. Senthil, A. Vishwanath, L. Balents, S. Sachdev, and M.P.A. Fisher, Science 303, 1490 (2004).

2 T. Senthil, L. Balents, S. Sachdev, A. Vishwanath, and M.P.A. Fisher, Phys. Rev. B 70, 144407 (2004).

3 T. Senthil, L. Balents, S. Sachdev, A. Vishwanath, and M.P.A. Fisher, J. Phys. Soc. Japan 74 suppl., 1 (2005).

4 J. Alicea, O.I. Motrunich, M. Hermele, and M.P.A. Fisher, Phys. Rev. B 72, 064407 (2005).

5 T. Senthil and M.P.A. Fisher, Phys. Rev. B 74, 064405 (2006).

6 A. Tanaka and X. Hu, Phys. Rev. B 74, 140407(R) (2006).

7 G.-Z. Liu, Phys. Rev. B 71, 172501 (2005).

8 R. Dillenschneider and J. Richert, Phys. Rev. B 73, 224443 (2006).

9 P. Ghaemi and T. Senthil, Phys. Rev. B 73, 054415 (2006).

10 I. O. Thomas and S. Hands, Phys. Rev. B 75, 134516 (2007).

11 A.W. Sandvik, Phys. Rev. Lett. 98, 227202 (2007).

12 R.G. Melko and R.K. Kaul, Phys. Rev. Lett. 100, 017203 (2008).

13 F.-J. Jiang, M. Nyfeler, S. Chandrasekharan, and U.-J. Wiese, J. Stat. Mech., P02009 (2008).

14 K. Krüger and S. Scheidl, Europhys. Lett. 74, 896 (2006).

15 A.B. Kuklov, M. Matsumoto, N.V. Prokof'ev, B.V. Svistunov, and M. Troyer, Phys. Rev. Lett. 101, 050405 (2008).

16 R. Coldea, D.A. Tennant, A.M. Tsvelik, and Z. Tylczynski, Phys. Rev. Lett. 86, 1335 (2001).

17 S. Yunoki and S. Sorella, Phys. Rev. Lett. 92, 157003 (2004).

18 A.E. Trumper, Phys. Rev. B 60, 2987 (1999).

19 Zheng Weihong, R.H. McKenzie, and R.P. Singh, Phys. Rev. B 59, 14367 (1999).

20 Zheng Weihong, J.O. Fjaerestad, R.R.P. Singh, R.H. McKenzie, and R. Coldea, Phys. Rev. Lett. 96, 057201 (2006).

21 C.H. Chung, J.B. Marston, and R.H. McKenzie, J. Phys.: Condens. Matter 13, 5159 (2001). 


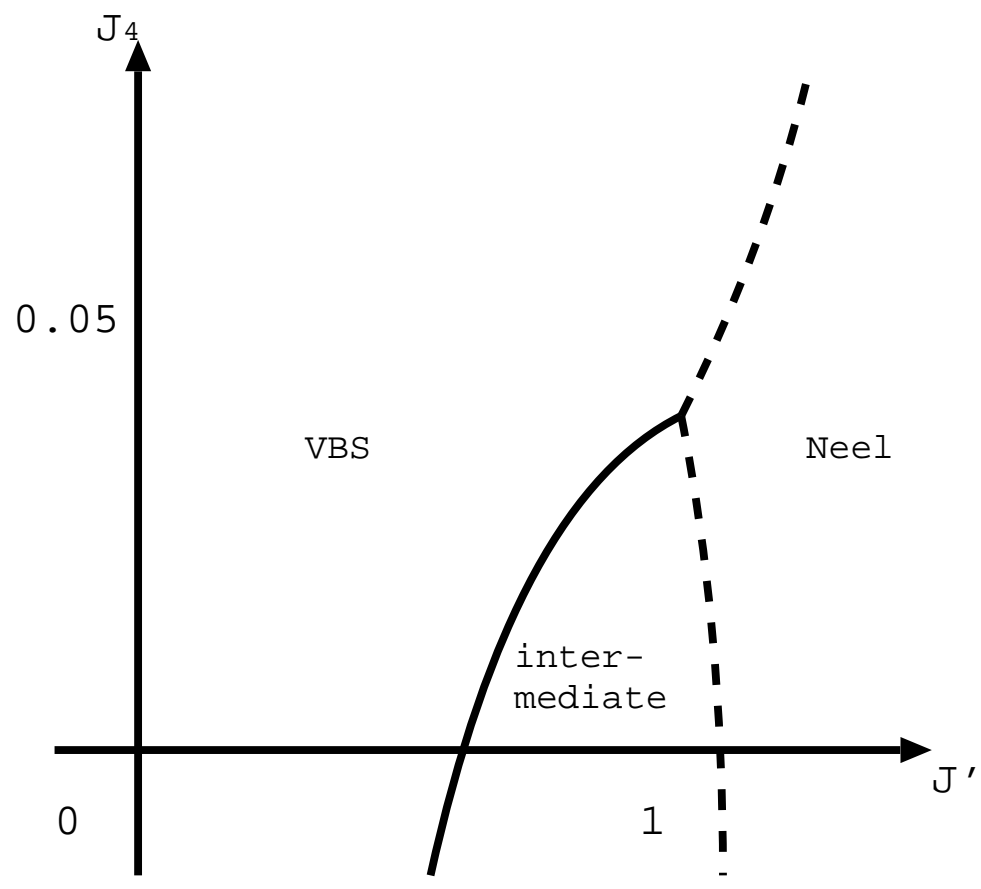

FIG. 1: A schematic phase diagram for the spatially anisotropic triangular antiferromagnet (1) is presented. The solid (dashed) line stands for the first- (second-) order phase boundary. Our concern is to realize a direct VBS-Néel transition by applying the ring exchange $J_{4}$, and analyze the singularity in the context of the deconfinement criticality. Concerning the singularities of the phase boundaries surrounding the intermediate phase, we follow the conclusion of Ref. 22 .

22 M.Q. Weng, D.N. Sheng, Z.Y. Weng, and R.J. Bursill, Phys. Rev. B 74, 012407 (2006).

23 G. Misguich, C. Lhuillier, B. Bernu, and C. Waldtmann, Phys. Rev. B 60, 1064 (1999).

24 B. Bernu, P. Lecheminant, C. Lhuillier, and L. Pierre, Phys. Rev. B 50, 10048 (1994).

25 J. Oitmaa and Zheng Weihong, Phys. Rev. B 54, 3022 (1996).

26 J. Sirker, Z. Weihong, O.P. Sushkov, and J. Oitmaa, Phys. Rev. B 73, 184420 (2006).

27 D. Poilblanc, A. Läuchli, M. Mambrini, and F. Mila, Phys. Rev. B 73, 100403(R) (2006).

28 P. Sindzingre, Phys. Rev B 69, 094418 (2004).

29 M. Mambrini, A. Läuchli, D. Poilblanc, and F. Mila, Phys. Rev. B 74, 144422 (2006).

30 K. Harada, N. Kawashima, and M. Troyer, J. Phys. Soc. Japan 76, 013703 (2007).

31 F.S. Nogueira, S. Kragset, and A. Sudbo, Phys. Rev. B 76, 220403(R) (2007).

32 M. Campostrini, M. Hasenbusch, A. Pelissetto, P. Rossi, and E. Vicari, Phys. Rev. B 65, 144520 (2002). 


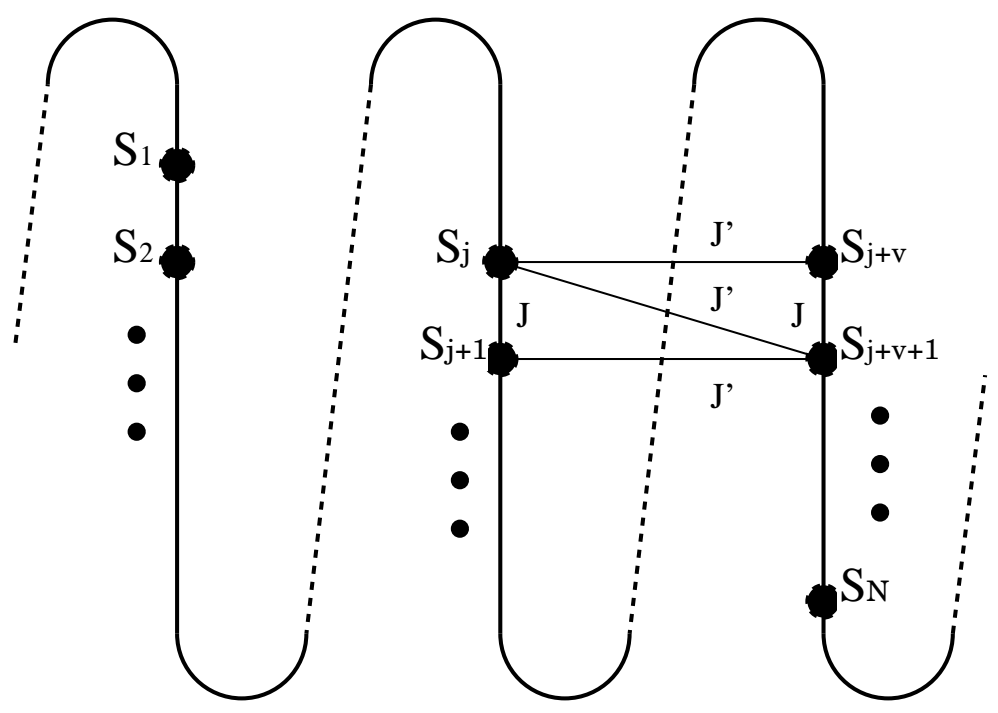

FIG. 2: A schematic drawing of the spin cluster under the screw-boundary condition for the spatially anisotropic triangular antiferromagnet (11) is presented. As indicated, the spins constitute a one-dimensional $(d=1)$ alignment $\left\{S_{i}\right\}(i=1,2, \ldots, N)$ via the longitudinal coupling $J$. The dimensionality is lifted to $d=2$ by the bridges over the $v$ th-neighbor pairs through the transverse coupling $J^{\prime}$. The screw pitch $v$ is given by Eq. (5) .

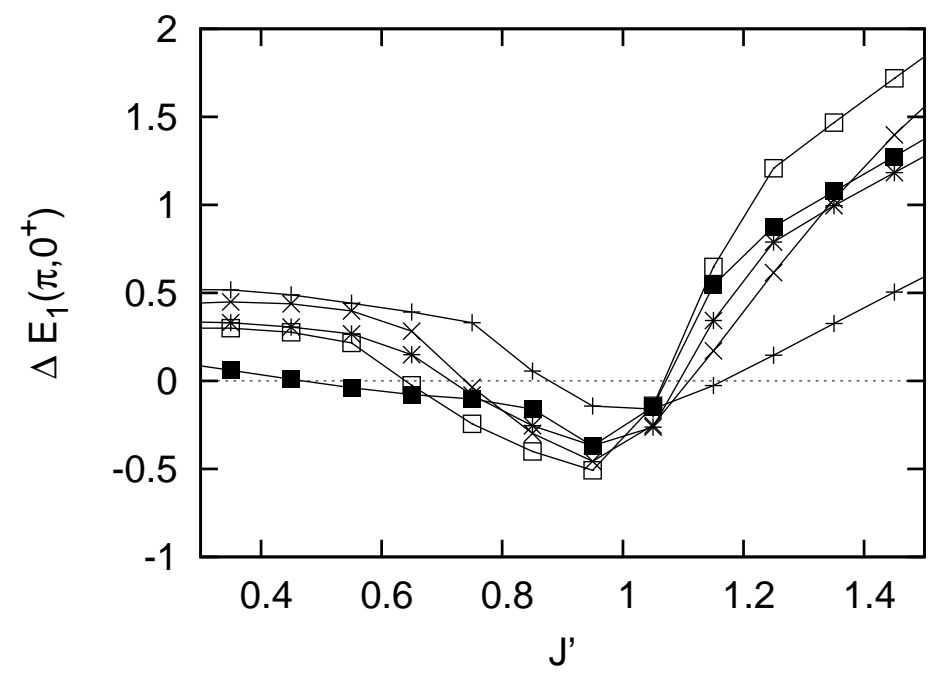

FIG. 3: The energy gap $\Delta E_{1}\left(\pi, 0^{+}\right)$, Eq. ([6) $)$, is plotted for $J_{4}=0$, various $J^{\prime}$, and $N=(+) 16,(\times)$ 20, (*) 24, ( $\square$ ) 28, and (ם) 32. A softening instability, $\Delta E_{1}<0$, takes place in the intermediate phase $0.65(15)<J^{\prime}<1.1(1)$. 


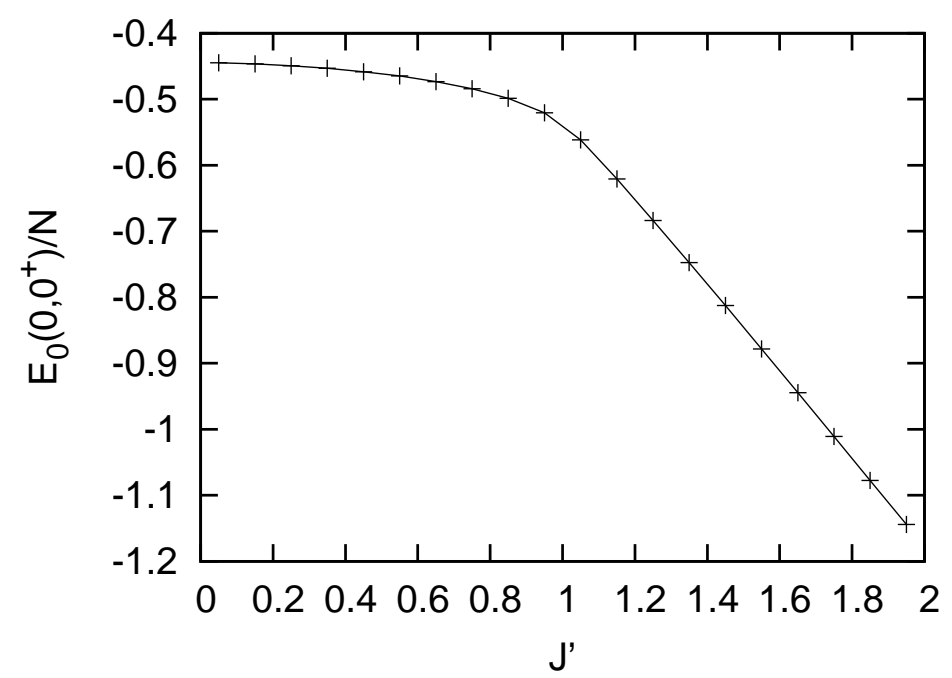

FIG. 4: The ground-state energy per unit cell, $E_{0}\left(0,0^{+}\right) / N$, with $N=32$ is presented for the same parameter range as that of Fig. 3. In the small- $J^{\prime}$ regime, the ground-state energy is close to the Bethe-ansatz solution, $E_{0} / N=-0.443 \ldots$, for the one-dimensional Heisenberg antiferromagnet.

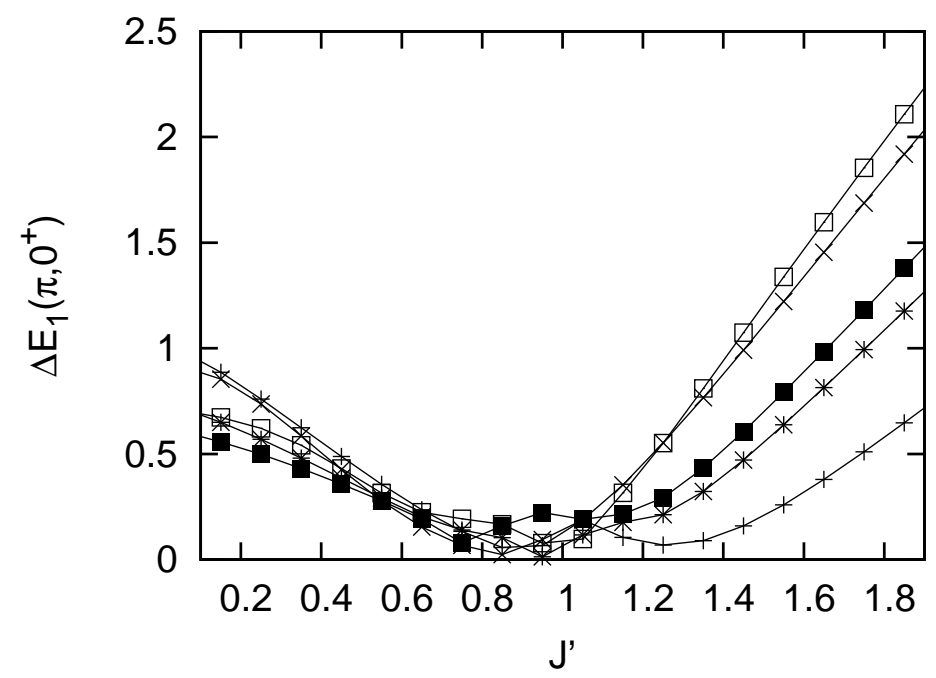

FIG. 5: The energy gap $\Delta E_{1}\left(\pi, 0^{+}\right)$, Eq. (6) , is plotted for $J_{4}=0.07$, various $J^{\prime}$, and $N=(+) 16$, $(\times) 20,(*) 24,(\square) 28$, and (ם) 32. Owing to the ring exchange, the softening instability, namely, $\Delta E_{1}<0$, does not occur any more. 


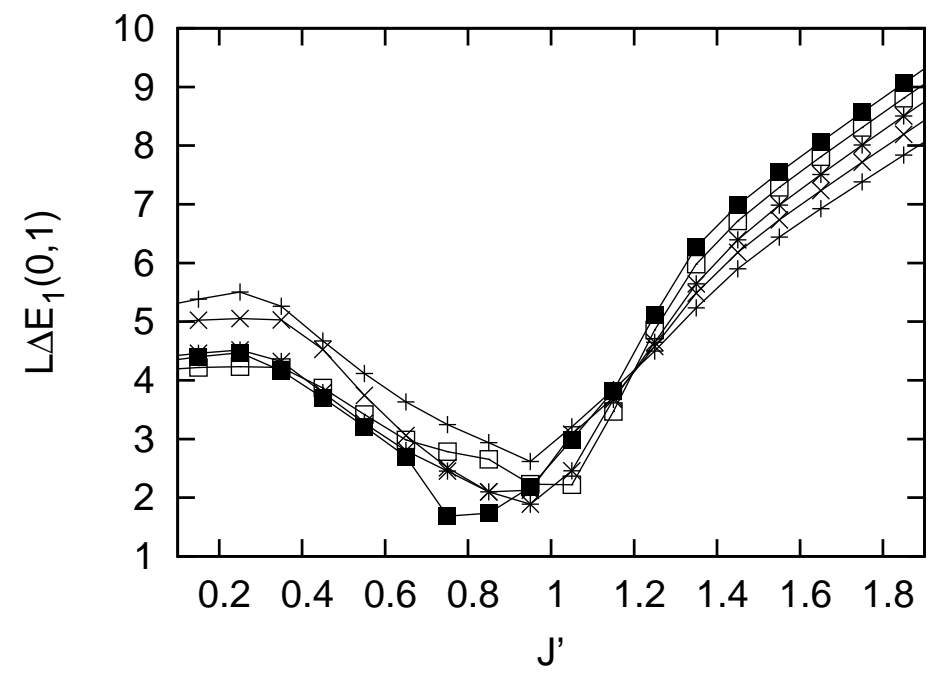

FIG. 6: The scaled triplet-excitation gap $L \Delta E_{1}(0,1)$ is plotted for $J_{4}=0.07$, various $J^{\prime}$, and $N=$ $(+)$ 16, (× 20, (*) 24, ( $\square) 28$, and $(\mathbf{\square}) 32$. A continuous transition takes place at $J_{c}^{\prime}=1.2$.

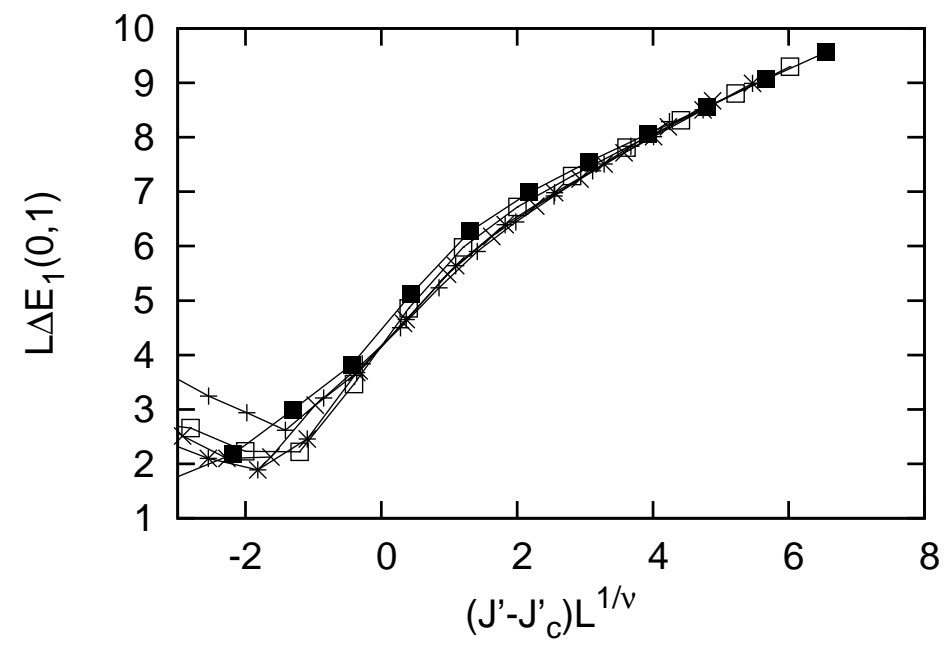

FIG. 7: The finite-size-scaling plot, $\left(J^{\prime}-J_{c}^{\prime}\right) L^{1 / \nu}-L \Delta E_{1}(0,1)$, is shown for $\nu=0.8, J_{c}^{\prime}=1.2$, $J_{4}=0.07$, various $J^{\prime}$, and $N=(+) 16,(\times) 20,(*) 24,(\square) 28$, and $(\mathbf{\square}) 32$. 


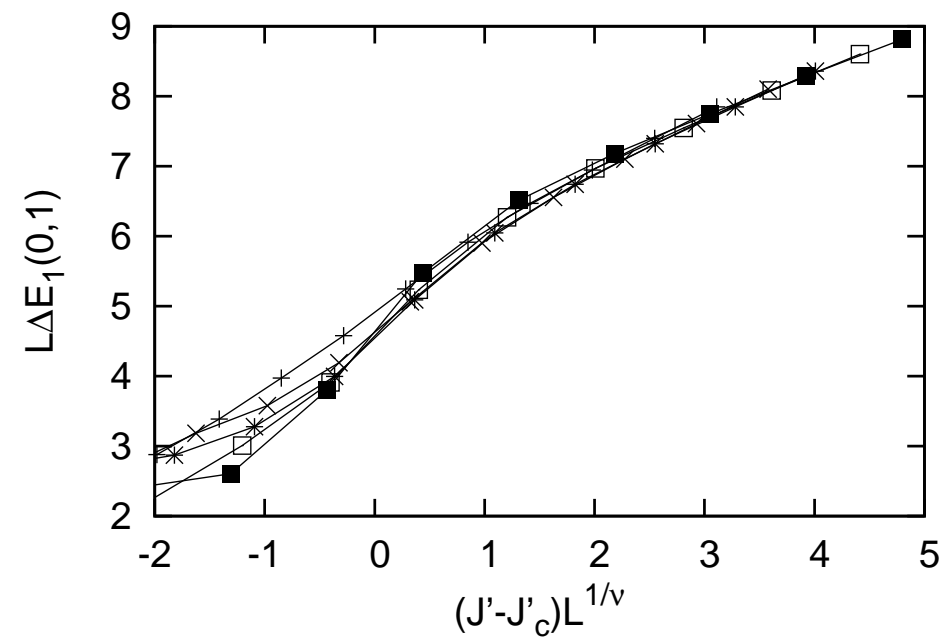

FIG. 8: The finite-size-scaling plot, $\left(J^{\prime}-J_{c}^{\prime}\right) L^{1 / \nu}-L \Delta E_{1}(0,1)$, is shown for $\nu=0.8, J_{c}^{\prime}=1.4$, $J_{4}=0.1$, various $J^{\prime}$, and $N=(+) 16,(\times) 20,(*) 24,(\square) 28$, and (匚) 32 . 